\title{
Fulfilment of Lighting Blinds Control System
}

\author{
Wen-Bin Lin ${ }^{1}$, Kao-Feng Yarn ${ }^{2}$ \\ ${ }^{1}$ Department of Electronic and Optoelectronic Application Engineering, ${ }^{2}$ Department of Aircraft Maintenance, \\ Far East University, Taiwan 744, ROC \\ Corresponding Author: Wen-Bin Lin
}

\begin{abstract}
This paper uses the characteristics of the 89C51 single chip to simulate the automatic control of the opening and closing of the blinds model by the automatic control of the ambient light intensity. Finally, the operating principle of the adopted electric circuits and the performance of the proposed device are validated by experimental results. It reveals that the operations of the proposed device coincide with the requirements.

The goal of this paper is that the blinds can automatically sense the outdoor brightness and adjust their opening and closing to achieve the effect of automatically adjusting the indoor brightness. Normally, the blinds must be manually rotated by the plastic tube on the blinds to adjust the angle of the blades on the blinds. Thus, it can change the brightness of the room. This article hopes that the blinds can detect the brightness given by the outside world to adjust and change the angle of its blades. It can sense the indoor light source and adjust the angle of the blinds during the day, and introduce daylight to achieve the effect of adjusting the indoor light.
\end{abstract}

Keywords: Lighting, Blind, 89C51 single chip, Light sensor, Stepper motors

\section{INTRODUCTION}

This article adapts the performance of 89C51 single chip to simulate the automatic control of the opening and closing of the blinds model by the automatic control of the ambient light intensity. The system architecture includes a set of 89C51 control circuits, a set of power processing circuits, a set of light sensors, a set of light level adjustment comparators, a set of action mode selection switches, a set of stepping motor drive circuits, a group of stepping motors, and a group of blind mechanism models. A group of power processing circuits to generate DC5V power supply for power processing circuits, single-chip control, stepper motors, light sensor, comparator, multi-program processing, to achieve automatic control technology ${ }^{[1-12]}$.

The 89C51 single chip is widely used in micro-machines, especially in engineering, it has become quite popular. Because 89C51 single-chip microprocessing machine has many advantages, such as powerful command function, large memory capacity, serial communication, Boolean algebra, etc. It allows us to design as we want, develop new products and product improvement.

89C51 single chip can play an important role in communication engineering, programmable controller, instrumentation engineering, and measurement engineering. With the popularity of personal computers, software development tools are fully supported, we can allow easily to use high-level $\mathrm{C}$ language to design general engineering application control programs on the $\mathrm{PC}$, and directly control hardware actions. Only in real applications, we often feel that we use a PC, but only do a little control, which is a bit overkill. It seems that with 89C51 single chip, it can be solved. Moreover, the single chip is small in size, simple to use, easily hardware wiring, low price, and electricity, it is a good choice. Choose a microcomputer not only consider the price level, as well as 
its work ability and ability to drive, so that the function of a microcomputer can be fully used to obtain cost-effective applications.

We take the control system of the lighting blinds control system as the research content of this implementation. In order to be effectively applied to the blinds to automatically sense the brightness of the outdoor, and adjust its own opening and closing to achieve the effect of automatically adjusting the indoor brightness.

\section{THEORETICAL FRAMEWORK}

The Block diagram of hardware composition is shown in Figure 1. The functions are depicted as follows:
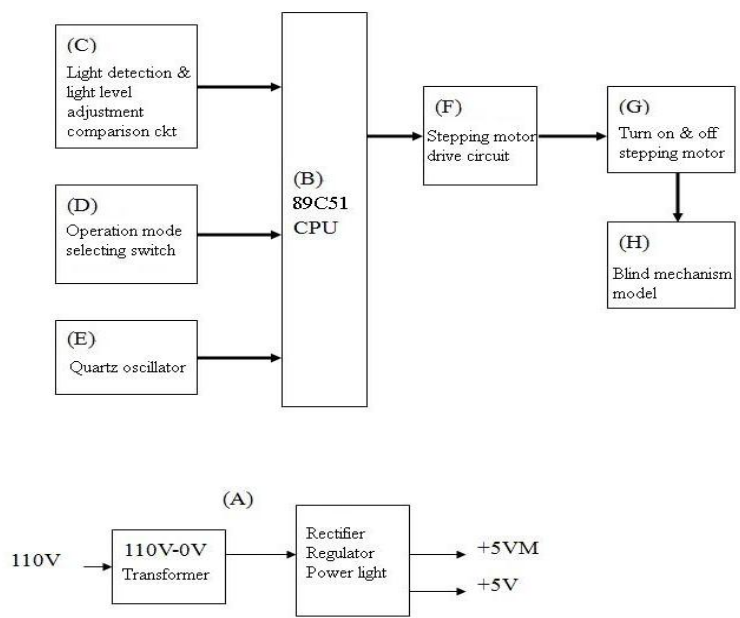

Figure 1: Block diagram of hardware composition

A. 8051 control circuit board function:

1. A set of 89C51 single-chip circuits are responsible for program control.

2. A set of power processing circuits.

3. A set of light sensors.

4. A set of light level adjustment comparators.

5. A group of operation mode selection switches.

6. A set of stepping motor drive circuit.

7. A set of stepping motor.

8. A set of blind mechanism model.

As shown in Figure 1, the principle of each circuit block is explained:

\section{(A) Power switch variable voltage power supply lamp circuit:}

AC110V input is provided by the grid power, through the switch, and then through the $110 \mathrm{Vac}$ to $15 \mathrm{Vac}$ transformer. After two sets of rectification, 7805 voltage regulator IC, DC5V DC power is available for IC use. And the other set of 7805 voltage regulator ICs, that is, DC5V DC power, used for stepping motor. When the DC5V power supplies, the LED power light is on.

\section{(B) 89C51 CPU circuit:}

89C51 is a 40 pin CPU, and its I/O pin has 32 pins.

1. The 40th pin is the VCC power input, and the 20th pin is the ground pin. RST pin $\left(9^{\text {th }}\right.$ pin), connect a $10 \mu F / 16 \mathrm{~V}$ capacitor and $2 \mathrm{~K}$ resistor, you can get the reset time of $10 \mu \times 2 k=20 \mathrm{~ms}$. This time period can avoid the situation that the power supply is not stable when the power is turned on and the program is disordered.

2. The 18th and 19th pins are the pins of the quartz oscillator, which provide the time pulse required for program execution.

3. The 29th, 30th, 31st pins are the external memory control pins. This article uses 8051 internal memory, so the 3 pins are not used, so they are connected to $10 \mathrm{k} \Omega$ and pulled to $5 \mathrm{~V}$.

4. The first PIN is the light detection and light level adjustment comparison input pin.

5. The 5th PIN is the input pin of the operation mode selection switch.

6. The 36th-39th PIN is the stepping motor drive output pin.

\section{(C) Light detection circuit:}

The light is detected by the photoresistor (the brighter the resistance, the lower the resistance value), and the voltage change caused by the light change is obtained according to the principle of partial pressure. The 50K VR can adjust the light start level, and then use the LM358 operational amplifier as a comparator to get $0 \mathrm{~V}$ (dark) or $5 \mathrm{~V}$ (bright) and input to the first PIN of 89C51. 
(D) Operation mode selection switch:

When the operation mode selection switch is ON, OV can be input to the 5th PIN of 89C51. When the operation mode selection switch is OFF, $5 \mathrm{~V}$ can be input to the 5th PIN of 89C51.

\section{(E) Quartz oscillator circuit:}

Through the quartz oscillator and the capacitive element, the oscillation provides the time pulse necessary for the program execution.

\section{(F) Stepping motor drive circuit:}

89C51 controls 4 transistors, when the corresponding coils in the stepping motor are turned on when they are sequentially $\mathrm{ON}$ (High). Each time a transistor is turned on, the stepping motor will take one step.

\section{(G) Turn on and off rotating stepping motor:}

It is a 4-phase stepping motor, which drives the blind mechanism through 89C51 control.

\section{(H) Blind mechanism model:}

The blinds that can be opened and closed by stepping motors are hand-made by woodworkers. Figure 2 and Figure 3 are system circuit diagrams.



Figure 2: System circuit diagram

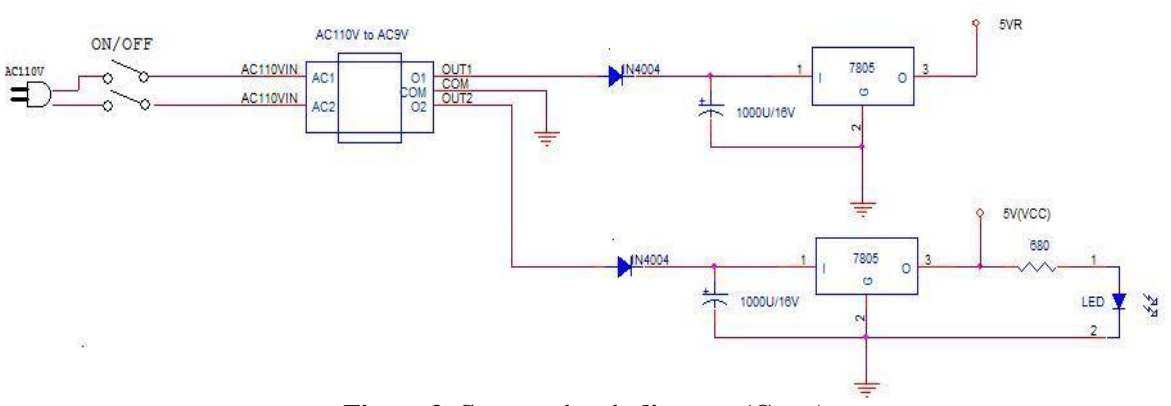

Figure 3: System circuit diagram (Cont.)

\section{MAIN FLOW CHART OF LIGHT CONTROL BLINDS SYSTEM PROGRAM}

Figure 4 is the main flow chart of light control blinds system program.

\section{FUNCTIONAL SPECIFICATIONS AND PRODUCTION RESULTS}

1. The main function of this system is the automatic control of the blind model switch by the automatic control of the ambient light intensity.
2. The system architecture includes a set of 89C51 control circuits, a set of power processing circuits, a set of light sensors, a set of light level adjustment comparators, a set of operation mode selection switches, a set of stepping motor drive circuits, and a set of stepping motors, and a set of blinder mechanism models (approximately $35 \mathrm{~cm} \times 30 \mathrm{~cm})$.

3. (1)When the operation mode selection switch is ON: If the light is weak, the 
blinds will automatically open. If the light is strong, the shutters will automatically close. (2)When the operation mode selection switch is OFF: If the light is weak, the shutters will automatically close. If the light is strong, the blinds will automatically open.

4. The opening and closing of the blind mechanism model is controlled by a set of stepping motor.

5. This fulfilment applies automatic control technologies such as stepping motors, light sensing, and potential comparators.

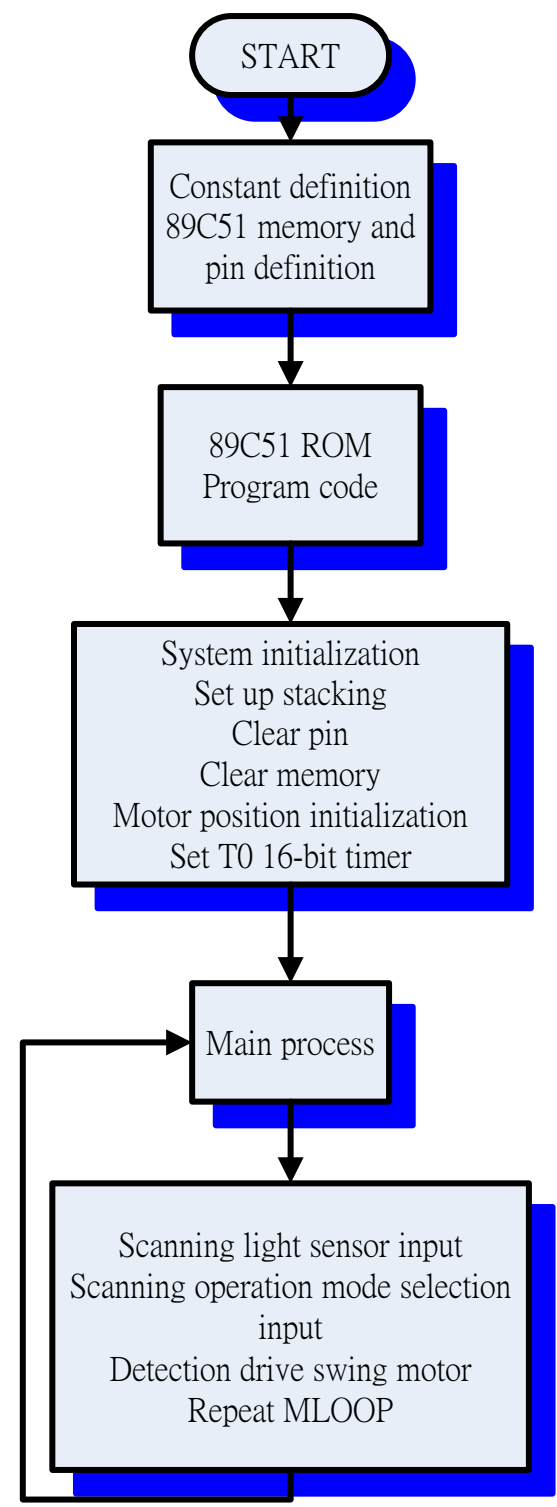

Figure 4: Main flow chart of light control blinds system program

This paper uses a photoresistor as the light sensor to sense the brightness.
Shown as in Figure 5, when the blinds are indoors and in a bright state, they are sensed by a photoresistor. The stepping motor adjusts the blade of the blinds to "close state". When the blinds are indoors and the brightness is not enough, the blinds will be opened as shown in Figure 6.

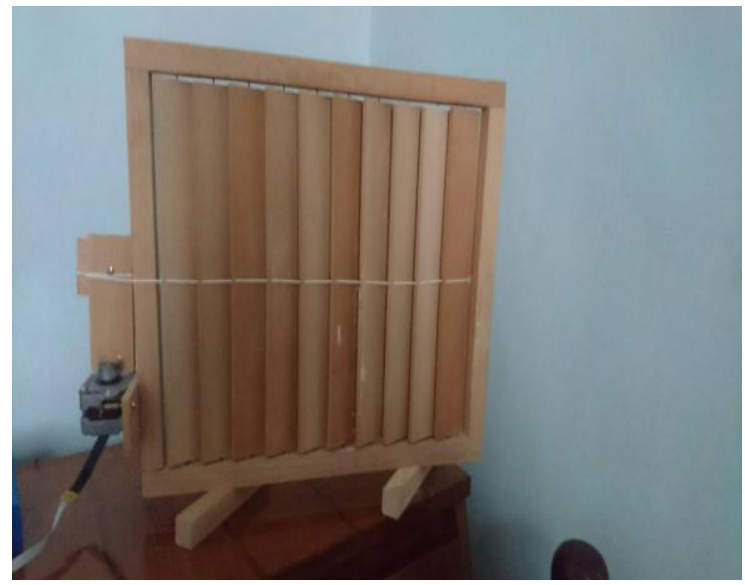

Figure 5: The blade of the blinds are closed

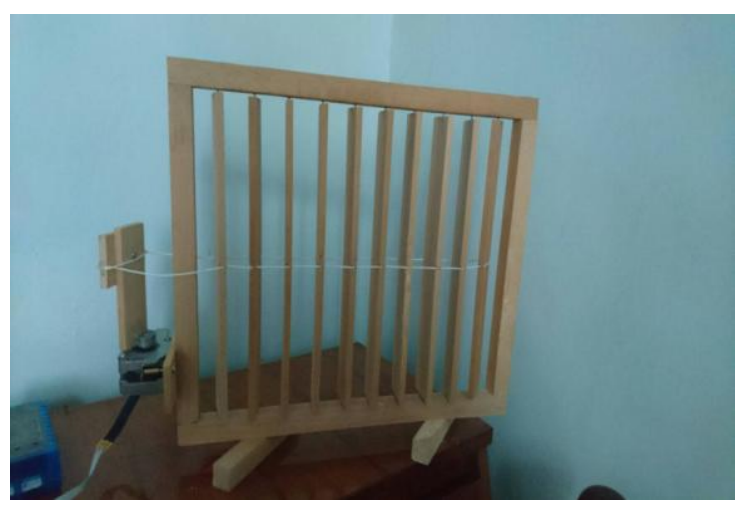

Figure 6: The blade of the blinds are opened

When the operation mode selection switch is OFF, the blinds become another mode, called "inverting control". As shown in Figure 6, when the photo resistor senses the light source, the blinds are opened. If the photo resistor cannot sense the light source, the blinds are closed. These are the two modes corresponding to the design of setting the light sensor of the blinds outdoors or indoors.

\section{CONCLUSION}

Figures 5 to 6 are photos of the hardware achievements of this paper. This paper implements a group of circuits that meet the above functions, and uses the results to verify the operation of the 
designed circuit and the functions that meet the requirements. It can be as an embryonic form reference for researchers engaged in related research.

\section{Acknowledgement: None}

\section{Conflict of Interest: None}

\section{Source of Funding: None}

\section{REFERENCES}

1. M.Y. Chen, A Single-Chip 8051 Implementation Entry Production Project, Wenkui Information Publishers, Taiwan, 2003.

2. Z.Y. Lu, 8051 Single-Chip Microcomputer Application, Gao Li Book Co., Ltd., Taiwan, 1999.

3. Z.Z. Chen, 8051 Single Chip Introduction and Practice, Rulin Book Company, Taiwan, 1995.

4. X.F. Wang, Principles and Applications of MCS-51 Single Chip Microcomputer (Practical Examples), Rulin Book Company, Taiwan, 1998.

5. M.C. Xu, 8051 Single-Chip Principle and Practice, Xinwenjing Development and Publishing Co., Ltd., Taiwan, 2000.
6. G.Y. Dai, Introduction to 8051 Single-Chip Microcomputer, Taiwan Fuwen Industrial Co., Ltd., Taiwan, 1995.

7. Z.Y. Sun, Handbook of Common Digital IC Materials, Quanhua Science and Technology Co., Ltd., Taiwan, 2001.

8. L.C. Chen, Principles And Key Points of Stepping Motor, Jianxing Publishing Co., Ltd., Taiwan, 1982.

9. S.M. Wu, Mechanical Design, Great China Book Company, Taiwan, 1980.

10. M.F. Yang, 8051 single-chip design practice, Jifeng Information Co., Ltd., Taiwan, 2003.

11. C.Y. Tsai, Original and Application of Single Chip Microcomputer 8051/8951 (Revised Third Edition), Published by Quanhua Publishing Co., Ltd., Taiwan, 2001.

12. Y.S. Guo, Development of Environmental Control System for Flower Cultivation Greenhouse, Master's Thesis of Department of Electrical Engineering, National Yunlin University of Science and Technology, Taiwan, 2009.

How to cite this article: Wen-Bin Lin, Kao-Feng Yarn. Fulfilment of lighting blinds control system. International Journal of Research and Review. 2021; 8(4): 126-130. DOI: https:// doi.org/10.52403/ijrr.20210418 